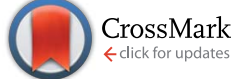

Cite this: J. Anal. At. Spectrom., 2017, 32, 393

Received 4th November 2016 Accepted 22nd December 2016

DOI: 10.1039/c6ja00402d

www.rsc.org/jaas

\section{Measurement of uranium-236 in particles by secondary ion mass spectrometry}

\author{
David S. Simons ${ }^{\star a}$ and John D. Fassett ${ }^{\mathrm{b}}$
}

The determination of the relative isotopic abundance by secondary ion mass spectrometry of ${ }^{236} U$ in uranium-containing material is complicated by the presence of ${ }^{235} \mathrm{U}^{1} \mathrm{H}^{+}$ions at the same nominal mass as the uranium isotopic peak. The net intensity of the ${ }^{236} \mathrm{U}$ signal is usually determined by a peakstripping procedure, whereby the ${ }^{235} \mathrm{U}^{1} \mathrm{H}^{+}$contribution is obtained by applying the ${ }^{238} \mathrm{U}^{1} \mathrm{H}^{+} / /^{238} \mathrm{U}^{+}$ratio to the ${ }^{235} \mathrm{U}^{+}$signal. The subtraction of one signal from another has consequences for the uncertainty of the final ${ }^{236} \mathrm{U}$ abundance determination that may be especially significant when the amount of sample is limited, as is the case with small uranium particles that are of great interest for nuclear safeguards. We have developed a model based on Poisson counting statistics to determine the effects of various parameters on the uncertainty of the ${ }^{236} \mathrm{U}$ abundance, including uranium enrichment level, hydride-toparent ratio, uranium mass consumed during analysis, single versus multichannel counting, and sample substrate composition. The model predictions have been successfully tested against experimental measurements of uranium oxide particles of both $3 \%$ and $90 \%$ enrichment in ${ }^{235} \mathrm{U}$.

\section{Introduction}

Secondary ion mass spectrometry (SIMS) is used routinely to measure the isotopic composition of uranium in particles for nuclear safeguard applications. ${ }^{1}$ When large-geometry SIMS instruments (LG-SIMS) are used for these measurements, almost all spectral interferences that would be problematic in smaller geometry SIMS instruments can be eliminated with little or no effect on instrument transmission, and the measurement of the relative abundances of ${ }^{234} \mathrm{U},{ }^{235} \mathrm{U}$, and ${ }^{238} \mathrm{U}$ is straightforward when instrumental mass fractionation is calibrated with suitable isotopic reference materials. ${ }^{1}$ This same conclusion does not apply for ${ }^{236} \mathrm{U}$ because the ${ }^{236} \mathrm{U}^{+}$ion that is the measured species of choice under oxygen ion bombardment is affected by a spectral interference from ${ }^{235} \mathrm{U}^{1} \mathrm{H}^{+}$that is separated in mass from ${ }^{236} \mathrm{U}^{+}$by only one part in 38000 . These two peaks could in principle be resolved in LG-SIMS instruments, but the resulting measurement would suffer from a severe signal reduction that is unacceptable when the sample is a micrometer-sized particle of very limited mass.

The uranium hydride ion fraction in SIMS is affected by several variables including the intrinsic hydrogen content of the sample, the sample chamber vacuum conditions, and the current density of the primary ion beam. Control of these variables can reduce the hydride fraction but the smallest practical level that is typically observed when uranium oxide particles are

${ }^{a}$ Material Measurement Laboratory, National Institute of Standards and Technology, Gaithersburg, MD 20899-8371, USA. E-mail: david.simons@nist.gov

${ }^{b}$ Dakota Consulting, Inc., 1110 Bonifant Street, Silver Spring, MD 20910, USA mounted on commonly used graphite substrates is in the range of $10^{-4}$. The conventional method to account for the uranium hydride interference issue in SIMS is to determine its contribution at $\mathrm{m} / \mathrm{z} 236$ by measuring the ${ }^{238} \mathrm{U}^{1} \mathrm{H}^{+} /{ }^{238} \mathrm{U}^{+}$ratio and assuming that the ${ }^{235} \mathrm{U}^{1} \mathrm{H}^{+} / 235 \mathrm{U}^{+}$ratio is identical. ${ }^{1}$ This assumption is reasonable because hydride ion formation is a chemical process that should affect all of the isotopes of an element in a similar manner. This approach also assumes that no ${ }^{239} \mathrm{Pu}^{+}$is present in the spectrum since it cannot be practically resolved from ${ }^{238} \mathrm{U}^{1} \mathrm{H}^{+}$and would otherwise create a bias in the correction procedure. Although in principle the ${ }^{234} \mathrm{U}^{1} \mathrm{H}^{+}$ signal would affect the measurement of ${ }^{235} \mathrm{U}^{+}$, in practice this situation can be ignored because the hydride correction is expected to be less than $0.01 \%$ of the ${ }^{235} \mathrm{U}^{+}$signal.

The effect of the presence of uranium hydride ions on the measurement of ${ }^{236} \mathrm{U}$ can be shown graphically with several examples. Fig. 1(a) displays a hypothetical plot of positive ion SIMS signals in the uranium mass region from example 1 , an isotopically natural uranium sample, with relative ion intensities plotted on a logarithmic scale. The uranium ion abundances are shown in black bars and the uranium hydride ions in cross-hatched bars. For this example, the hydride ion fraction is assumed to be $10^{-3} \cdot{ }^{236} \mathrm{U}$ is produced by thermal neutron capture of ${ }^{235} \mathrm{U}$. In uranium ore some thermal neutrons are present due to spontaneous fission, resulting in a relative isotopic abundance of ${ }^{236} \mathrm{U}$ on the order of $10^{-10}, 2$ which is well below the detectability of conventional SIMS measurements. Therefore, the only measurable signal at $\mathrm{m} / \mathrm{z} 236$ for this example is ${ }^{235} \mathrm{U}^{1} \mathrm{H}^{+}$at a relative abundance of $7.2 \times 10^{-6}$ with respect to the total $\mathrm{U}$ signal. 

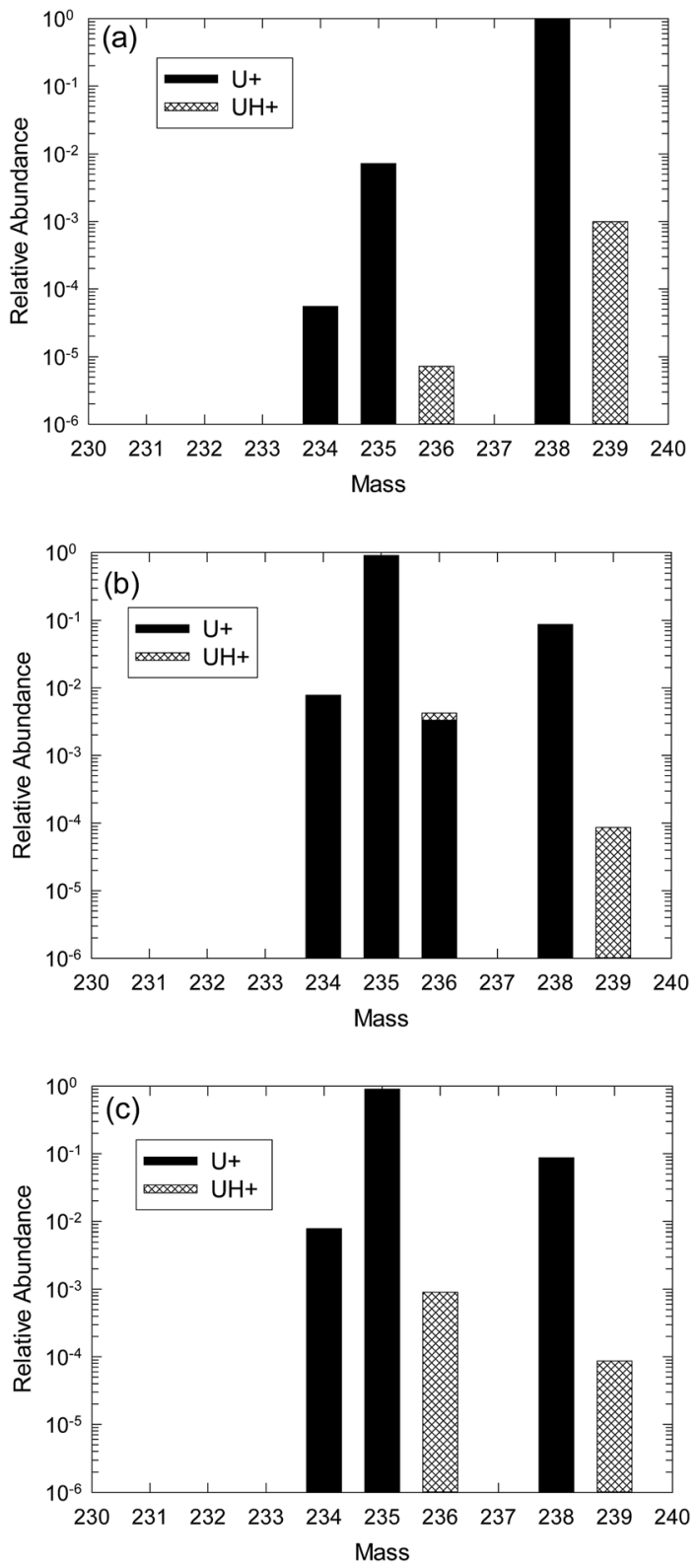

Fig. 1 (a) Graph of $\mathrm{U}^{+}$and $\mathrm{UH}^{+}$isotopic abundances for natural uranium with an assumed hydride fraction of $10^{-3}$; (b) same as (a) for $90 \%$ enriched uranium with $0.33 \%{ }^{236} \mathrm{U}$; (c) same as (b) for $90 \%$ enriched uranium with no ${ }^{236} \mathrm{U}$.

Example 2 demonstrates the effect of hydride production on highly-enriched recycled uranium that contains a significant amount of ${ }^{236} \mathrm{U}$. The presence of measureable ${ }^{236} \mathrm{U}$ that is well above the level in uranium ore is an indicator that the uranium was exposed to a significant neutron fluence, usually in a nuclear reactor, during its history. Fig. 1(b) shows a plot of relative abundances for CRM U900 (ref. 3) that is enriched to 90 atom percent in ${ }^{235} \mathrm{U}$ and also contains about 0.33 atom percent of ${ }^{236} \mathrm{U}$. Again a hydride fraction of $10^{-3}$ is assumed. In this case the hydride contribution at $\mathrm{m} / \mathrm{z} 236$ at a relative abundance of $9 \times 10^{-4}$ is much larger than for natural uranium but it contributes only about $25 \%$ additional signal to the actual ${ }^{236} \mathrm{U}$ signal in this material.
Example 3 is a material similar to U900, but containing only the very low natural level of ${ }^{236} \mathrm{U}$, as would be the case in an enrichment scenario that involved only natural uranium feed. This situation is depicted in Fig. 1(c). The amount of ${ }^{235} \mathrm{UH}$ is the same as in Fig. 1(b) but the relative uncertainty of the calculated amount of ${ }^{236} \mathrm{U}$ will be much larger than in the previous case because the net amount after the hydride correction will be much smaller (essentially zero). The correction in this case is more problematic than for natural uranium both because the hydride signal at $\mathrm{m} / \mathrm{z} 236$ is more than 100 times larger and because the signal at $\mathrm{m} / \mathrm{z} 239$ that is used for the correction is about 10 times smaller, thus creating a larger statistical uncertainty.

It is useful to understand the level of uncertainty with which the net ${ }^{236} \mathrm{U}$ abundance can be determined by SIMS in a particular circumstance, and what steps can be taken to minimize that uncertainty. To this end we have developed a mathematical model of the SIMS measurement process.

The equation to determine the ${ }^{236} \mathrm{U}$ content at $\mathrm{m} / \mathrm{z} 236$ relative to the reference isotope of ${ }^{235} \mathrm{U}$ based upon the procedure mentioned above is given as follows:

$$
\frac{{ }^{236} \mathrm{U}}{{ }^{235} \mathrm{U}}=\frac{{ }^{236} \mathrm{U}+{ }^{235} \mathrm{UH}}{{ }^{235} \mathrm{U}}-\frac{{ }^{238} \mathrm{UH}}{{ }^{238} \mathrm{U}} .
$$

When the reference isotope is ${ }^{238} \mathrm{U}$ the following equation is used:

$$
\frac{{ }^{236} \mathrm{U}}{{ }^{238} \mathrm{U}}=\frac{{ }^{236} \mathrm{U}+{ }^{235} \mathrm{UH}}{{ }^{238} \mathrm{U}}-\frac{{ }^{238} \mathrm{UH}}{{ }^{238} \mathrm{U}} \times \frac{{ }^{235} \mathrm{U}}{{ }^{238} \mathrm{U}} .
$$

Eqn (2) was also presented in ref. 1. In both eqn (1) and (2) all of the ratios on the right side can be measured experimentally.

The uncertainties in the net ${ }^{236} \mathrm{U}$ ratios can be derived by a propagation-of-errors approach from the above equations, where the terms are assumed to be statistically independent. This assumption will generally be valid if the denominators of the ratios in eqn (1) and (2) are much larger than the numerators, i.e., the reference isotope is the major isotope. When ${ }^{235} \mathrm{U}$ is the reference isotope, the equation for the variance of the ${ }^{236} \mathrm{U} /{ }^{235} \mathrm{U}$ ratio is:

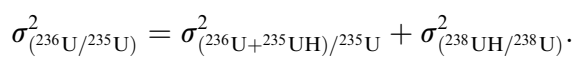

When the reference isotope is ${ }^{238} \mathrm{U}$, the corresponding equation is:

$$
\begin{aligned}
\sigma_{\left({ }^{236} \mathrm{U} /{ }^{238} \mathrm{U}\right)}^{2}= & \sigma_{\left({ }^{236} \mathrm{U}+{ }^{235} \mathrm{UH} /{ }^{238} \mathrm{U}\right)}^{2}+\sigma_{\left({ }^{235} \mathrm{U} /{ }^{238} \mathrm{U}\right)}^{2} \times\left(\frac{{ }^{238} \mathrm{UH}}{{ }^{238} \mathrm{U}}\right)^{2} \\
& +\sigma_{\left({ }^{238} \mathrm{UH} / 238 \mathrm{U}\right)}^{2} \times\left(\frac{{ }^{235} \mathrm{U}}{{ }^{238} \mathrm{U}}\right)^{2} .
\end{aligned}
$$

The detection limit for the measurement of ${ }^{236} \mathrm{U}$ by SIMS in uranium oxide particles was calculated recently by Ranebo et al. for a specific set of conditions. ${ }^{1}$ One condition assumed that 0.1 $\mathrm{pg}$ of uranium was consumed in the SIMS measurement. A second condition assumed that $1 \mathrm{pg}$ was consumed, corresponding to $2.5 \times 10^{9}$ atoms, an amount contained in a pure spherical $\mathrm{UO}_{2}$ particle with a diameter of about $0.6 \mu \mathrm{m}$. They 
also assumed a $1 \%$ efficiency for converting uranium atoms into detected ions and a hydride formation ratio of $8 \times 10^{-4}$ based on their experience with typical measured values for high performance magnetic sector SIMS instruments. They then calculated the ${ }^{236} \mathrm{U}$ detection limit as a function of enrichment level by a Monte Carlo method based on eqn (2) above with the definition of the detection limit being 3 times the standard deviation of the background. By this definition there is only a $1 \%$ probability of a false positive decision that ${ }^{236} \mathrm{U}$ is present if a measured value is at the detection limit. In this case the background was assumed to be the net ratio calculated in eqn (2) above when no ${ }^{236} U$ is actually present, with the uncertainties in the measured ratios determined by Poisson counting statistics and a small detector noise component. The calculations assume that a multicollector detection system such as the one available on the Cameca IMS-1280† is used to capture the counts of all of the uranium and relevant hydride species simultaneously. The results of the calculations by Ranebo et al. show that the ${ }^{236} \mathrm{U}$ detection limit in atom percent for consumption of $1 \mathrm{pg}$ of uranium during analysis by SIMS is a strong function of the enrichment level as a consequence of the hydride correction, rising from less than $0.0002 \%$ for natural uranium to about $0.005 \%$ for $90 \%$ enriched particles.

In the remainder of this paper we extend the approach of Ranebo et al. with a modified method of calculating detection limits, investigate the effects of various parameters on the measurement uncertainty and detection limit of ${ }^{236} U$ in particles by SIMS and compare our calculations with experimental measurements.

\section{Analytical calculations of ${ }^{236} \mathrm{U}$ detection limit and uncertainty}

We have developed an analytical approach based on eqn (1) and (3) for enrichments greater than $50 \%$ in ${ }^{235} \mathrm{U}$ and eqn (2) and (4) for enrichments less than $50 \%$. The calculations are made in an Excel spreadsheet and allow the following parameters to be varied:

(a) Uranium isotopic composition, fixing ${ }^{234} \mathrm{U} /{ }^{235} \mathrm{U}=0.01$ and summing all $\mathrm{U}$ abundances to $100 \%$.

(b) Total number of $\mathrm{U}$ atoms consumed, fixing the SIMS utilization efficiency at $1 \%$, consistent with experimental measurements. ${ }^{\mathbf{1 , 4}}$

(c) Hydride ratio.

(d) Single or multichannel detection; if single channel detection is assumed, $\mathrm{m} / \mathrm{z} 234,236$ and 239 are each assumed to be monitored for $25 \%$ of the time and $m / z 235$ and 238 for $12.5 \%$; settling time between species is ignored for simplicity; if multichannel detection is assumed, all species are monitored for $100 \%$ of the time.

\footnotetext{
$\uparrow$ Certain commercial equipment, instruments, or materials are identified in this paper to specify the experimental procedure adequately. Such identification is not intended to imply recommendation or endorsement by the National Institute of Standards and Technology, nor is it intended to imply that the materials or equipment identified are necessarily the best available for the purpose.
}

(e) The detector noise is assumed to make a negligible contribution to the measured signals. In our experience the noise level of pulse-counting electron multiplier detection systems can be routinely maintained below 0.003 counts per $\mathrm{s}$.

The calculation proceeds as follows. After fixing the isotopic composition, number of $\mathrm{U}$ atoms, hydride ratio and detection mode, the numbers of ions detected at $\mathrm{m} / \mathrm{z} 234,235,236,238$ and 239 are calculated. Either ${ }^{235} \mathrm{U}$ or ${ }^{238} \mathrm{U}$ is selected as the reference species depending on which is more abundant, and isotopic ratios are calculated, including ${ }^{236} \mathrm{U}$ from eqn (1) or (2). Ratio uncertainties are calculated based on Poisson counting statistics. The uncertainty of the ${ }^{236} \mathrm{U}$ ratio is calculated from either eqn (3) or (4). Finally, the isotopic ratios are converted to atom percents, and the atom percent uncertainties are calculated from the isotopic ratio uncertainties according to formulas that have been previously derived. ${ }^{5,6}$ For this final step it is important that the reference isotope for the ratios is the most abundant one to minimize errors due to covariance effects.

As an initial test, the detection limit for ${ }^{236} \mathrm{U}$ was calculated by this procedure for a range of enrichments using the same assumptions as were used by Ranebo et al. for the data in their Fig. 11(b) (1 pg case) except for the lack of a detector noise component. The graph of our data appears to be visually identical to theirs, validating the present approach. In the following sections we investigate the effects of several parameters on the ${ }^{236} \mathrm{U}$ detection limit.

\section{Effect of hydride ratio when ${ }^{236} \mathrm{U}$ is not present}

In this section we assume that $10^{10}$ atoms of $U$ are consumed in the SIMS measurement, equivalent to a mass of about $4 \mathrm{pg}$ and a corresponding pure $\mathrm{UO}_{2}$ particle diameter of about $0.9 \mu \mathrm{m}$. A single pulse-counting detector rather than a multichannel detector is assumed because the former is more widely applicable. Calculations were made for three different hydride ratios, $10^{-3}, 10^{-4}$ and $10^{-5}$, spanning the experimentally observed range of uranium hydride ion ratios.

The results of these detection limit calculations are shown in Fig. 2 for the 3 hydride ratios, where the ${ }^{236} \mathrm{U}$ detection limit in atom percent has been plotted on a logarithmic scale. The detection limit decreases by a factor of 10 when the hydride ratio decreases by a factor of 100 ; that is, the detection limit scales as the square root of the hydride ratio. If we consider a detection limit of 0.0001 atom percent as a benchmark for determining whether ${ }^{236} \mathrm{U}$ is present in a particle, then this limit cannot be achieved by SIMS with a hydride ratio of $10^{-3}$ while consuming $10^{10}$ atoms of $U$ unless the particle is highly depleted in ${ }^{235} \mathrm{U}$. The ${ }^{235} \mathrm{U}$ enrichment must be below $3 \%$ to achieve this benchmark if the hydride ratio is $10^{-4}$, and it must be below $20 \%$ if a hydride ratio of $10^{-5}$ can be achieved. For a fixed ${ }^{235} \mathrm{U}$ enrichment of $93 \%$, Fig. 3 shows the ${ }^{236} \mathrm{U}$ detection limit as a continuous function of the hydride ratio, illustrating the square-root relationship. For a hydride ratio of $10^{-3}$ the detection limit is 0.0074 atom percent, falling to 0.0023 atom 


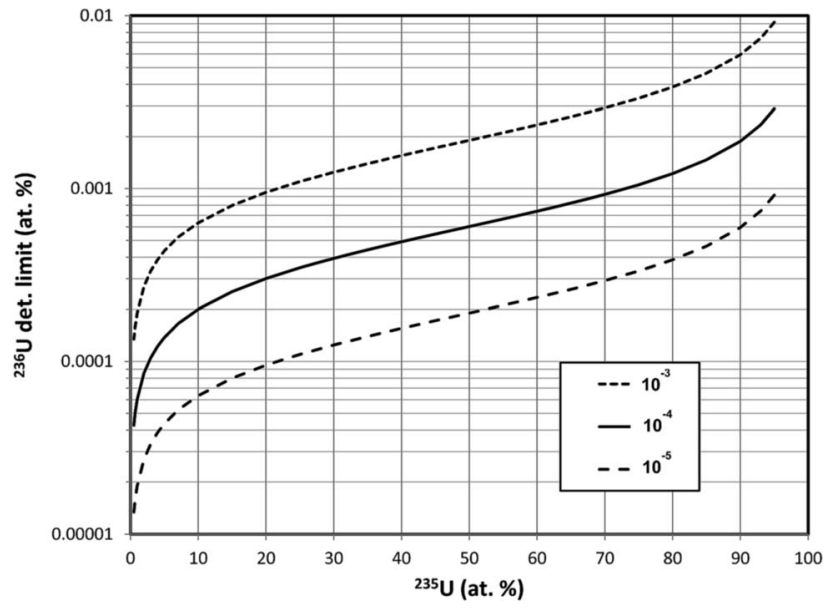

Fig. 2 Calculated detection limits for ${ }^{236} U$ as a function of enrichment for three different hydride ion ratios. Calculation assumes $10^{10}$ atoms consumed, single-channel peak-switching, 1\% SIMS efficiency.

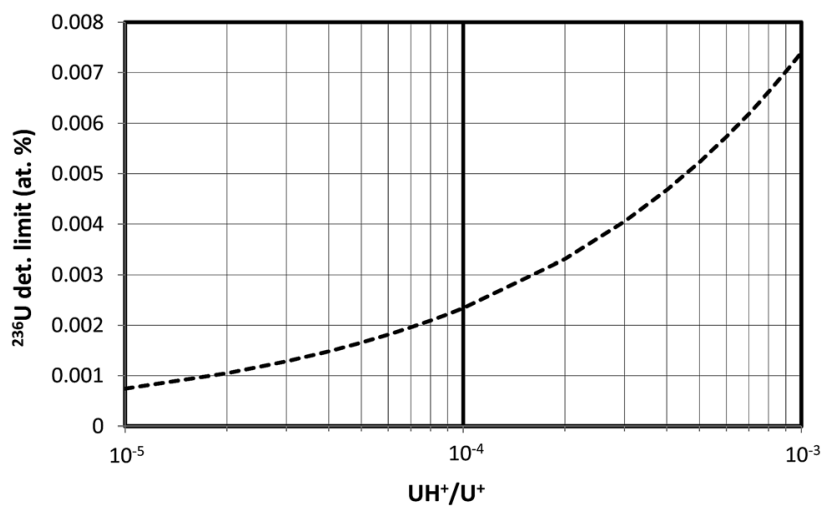

Fig. 3 Calculated ${ }^{236} U$ detection limit for total consumption of a $93 \%$ enriched particle as function of hydride ratio. Particle contains $10^{10}$ atoms of $U$ that are counted by peak-switching in a single-channel detector with $1 \%$ overall efficiency. Detection limit scales with the square root of the hydride ratio.

percent for a ratio of $10^{-4}$ and to 0.00074 atom percent for a ratio of $10^{-5}$. To achieve a detection limit of 0.0001 atom percent for this example a hydride ratio below $2 \times 10^{-7}$ would be required.

\section{Effect of uranium mass consumed}

The effect of consumed mass on the ${ }^{236} \mathrm{U}$ detection limit was investigated by holding other parameters constant. The same conditions as in the previous section were assumed, with a fixed hydride ratio of $10^{-4}$. Fig. 4 shows the effect of particle mass, plotted as equivalent diameter of a $\mathrm{UO}_{2}$ particle, on the detection limit for a $93 \%$ enriched particle. $\mathrm{U}_{3} \mathrm{O}_{8}$ particles of equivalent mass would have $11 \%$ larger diameters as a result of their lower density. Since the detection limit is controlled by the Poisson statistics of the $\mathrm{m} / \mathrm{z} 236$ and $\mathrm{m} / \mathrm{z} 239$ counts, it scales as the inverse square root of the mass, or as the inverse diameter to the $3 / 2$ power. In this example the detection limit does not fall

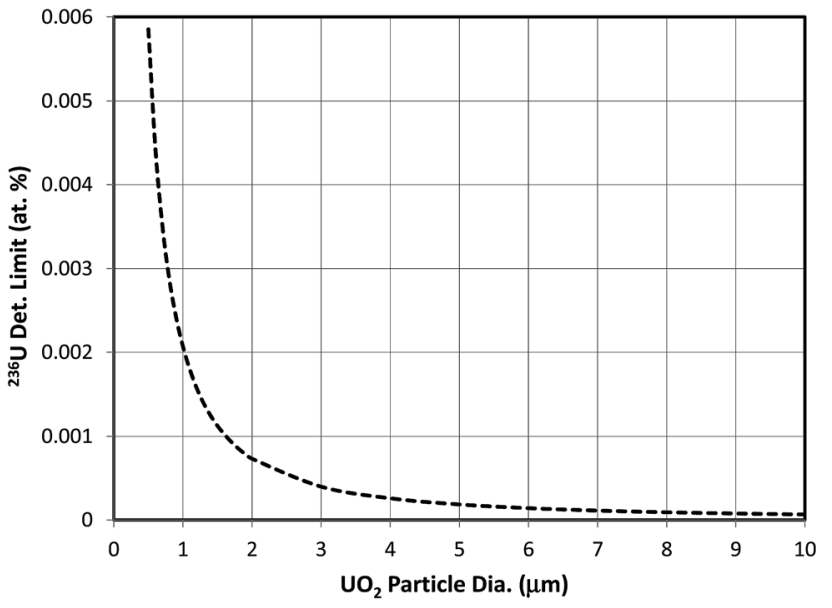

Fig. 4 Calculated ${ }^{236} U$ detection limit for total consumption of a $93 \%$ enriched particle as function of particle diameter for peak-switching in a single-channel detector with $1 \%$ overall efficiency. Detection limit scales with the inverse square root of the particle mass.

below 0.0001 atom percent unless the diameter is greater than $7.5 \mu \mathrm{m}$. This size of particle contains more than 400 times the mass of a $1 \mu \mathrm{m}$ particle. The likelihood of finding such a large particle in an environmental sample is very small, and it would require an inordinately long time to sputter that much material in a SIMS experiment while maintaining high ion transmission and keeping the major isotope count rate below $10^{6}$ counts per $\mathrm{s}$ to avoid significant counting losses due to pulse overlap in the pulse-counting system. The count-rate limitation could be avoided and the data could be acquired faster if the signals from the major $\mathrm{U}$ isotopes were recorded with Faraday cups.

\section{Effect of hydride ratio when ${ }^{236} \mathrm{U}$ is present}

When a significant amount of ${ }^{236} \mathrm{U}$ is actually present in a sample the uncertainty (standard deviation) of its abundance determination is of interest rather than the detection limit, which is relevant when little or no ${ }^{236} \mathrm{U}$ is present. To calculate this uncertainty we take the square root of the variance determined by eqn (3) or (4). This uncertainty has been calculated as a function of hydride ratio for three different uranium isotopic reference materials: CRM U010 (1.0037 atom percent ${ }^{235} \mathrm{U}$, 0.00681 atom percent ${ }^{236} \mathrm{U}$ ), CRM U030a (3.0404 atom percent ${ }^{235} \mathrm{U}, 0.000599$ atom percent ${ }^{236} \mathrm{U}$ ) and CRM U900 (90.196 atom percent ${ }^{235} \mathrm{U}, 0.3327$ atom percent ${ }^{236} \mathrm{U}$ ). ${ }^{3}$ As in the previous section, particles containing $10^{10}$ atoms of uranium, $1 \%$ efficiency and a single detector are assumed. The results of the calculations are plotted in Fig. 5. For all three cases the uncertainty reaches a limiting value for decreasing hydride ratios. This behavior is a consequence of the contribution of the ${ }^{236} \mathrm{U}$ term in eqn (3) or (4) which remains even when the terms involving the hydride ratio become negligible. For purposes of comparison we arbitrarily define a practical hydride ratio threshold as the hydride ratio where the uncertainty is $20 \%$ greater than its minimum value, so a ratio below that threshold 

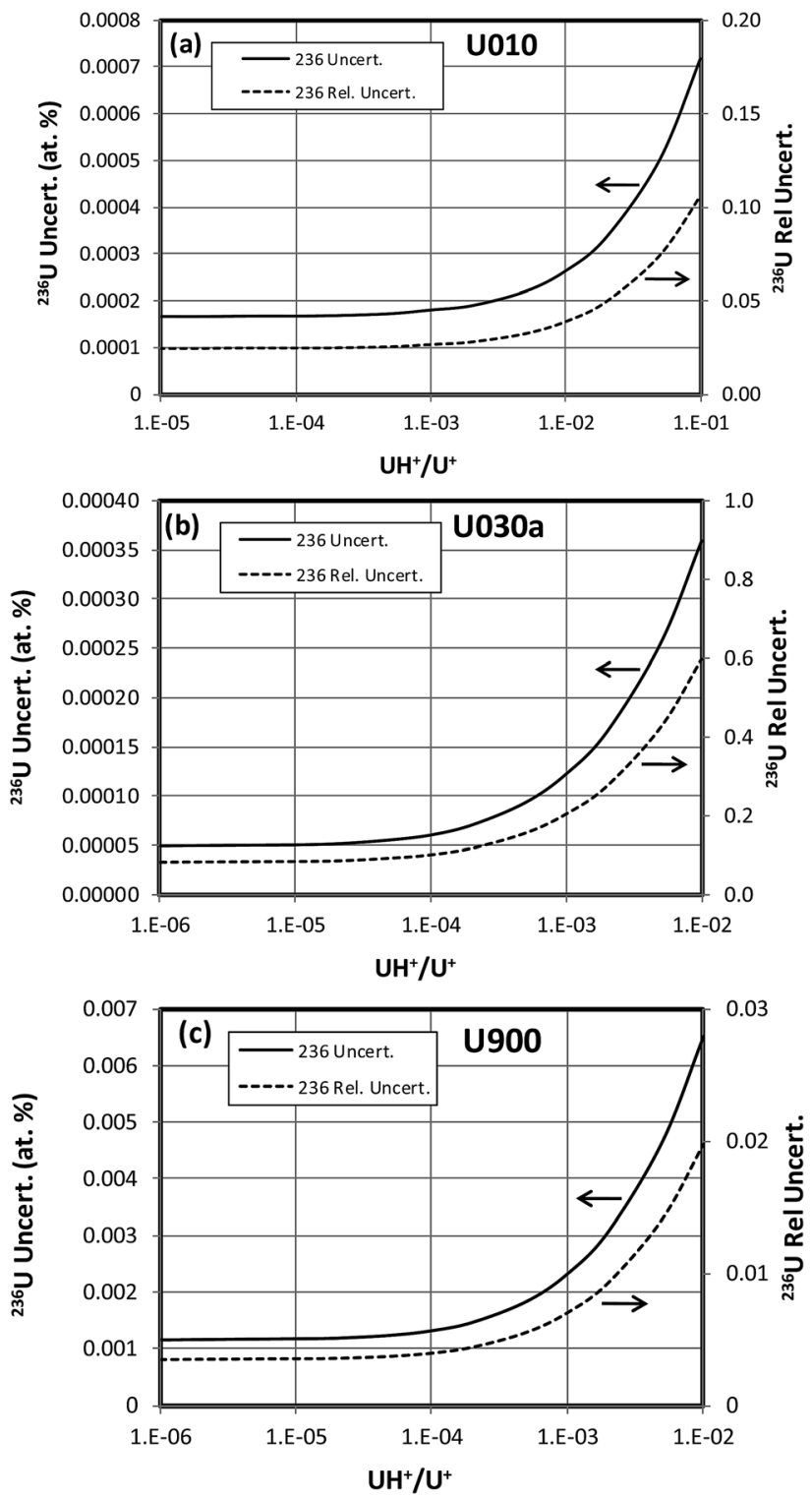

Fig. 5 Model calculations for absolute and relative uncertainties of ${ }^{236} \mathrm{U}$ isotopic abundance as a function of hydride ratio for particles of (a) CRM U010; (b) CRM U030a; (c) CRM U900. Arrows point to axes that apply to each curve.

wouldn't make much improvement in the ${ }^{236} \mathrm{U}$ uncertainty. For these three examples the ratio thresholds are $3 \times 10^{-3}, 1 \times 10^{-4}$ and $1.5 \times 10^{-4}$ respectively. Although the ${ }^{236} \mathrm{U}$ uncertainties depend on the size of the particle and the number of uranium ions detected, the hydride thresholds do not. Therefore, it appears that there is little value in achieving hydride ratios below $10^{-4}$ when a significant amount of ${ }^{236} \mathrm{U}$ is present.

The ${ }^{236} \mathrm{U}$ abundance level has an effect on its absolute uncertainty, the effect being stronger when the hydride ratio is low. Fig. 6 shows the results of calculations of the absolute and relative uncertainties (standard deviations) of ${ }^{236} \mathrm{U}$ abundance for $93 \%{ }^{235} \mathrm{U}$ enrichment, $10^{10}$ atoms and a $10^{-4}$ hydride ratio as the ${ }^{236} \mathrm{U}$ relative abundance varies from $0 \%$ to $1 \%$. The absolute uncertainty increases from about 0.0009 atom percent with no

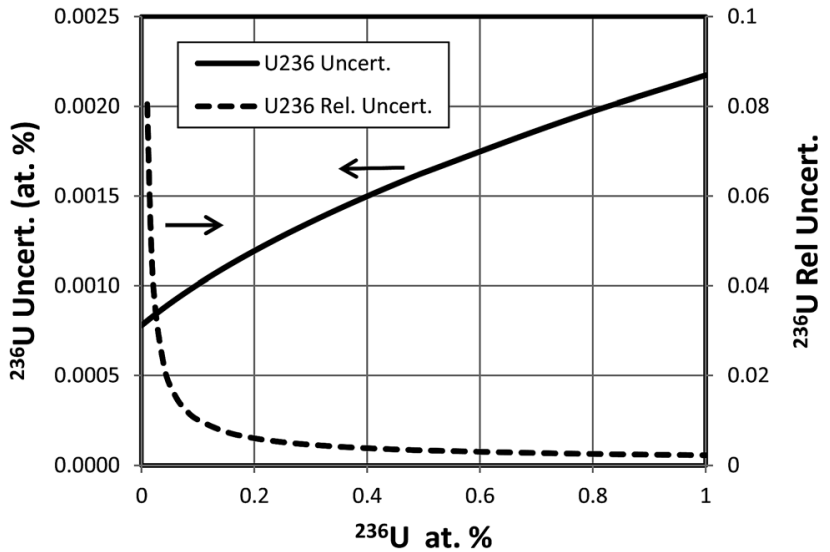

Fig. 6 Absolute and relative uncertainties for different levels of ${ }^{236} \mathrm{U}$ abundance in $93 \%$ enriched particle. Additional assumptions described in text.

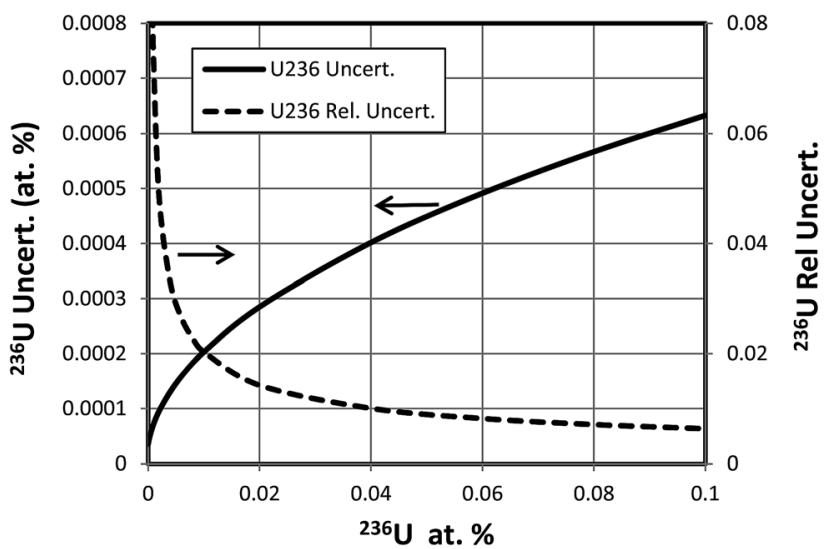

Fig. 7 Absolute and relative uncertainties for different levels of ${ }^{236} U$ abundance in $3 \%$ enriched particle. Additional assumptions described in text.

${ }^{236} \mathrm{U}$ present to about 0.002 atom percent with $1 \%{ }^{236} \mathrm{U}$ whereas the relative uncertainty is less than $1 \%$ when the ${ }^{236} \mathrm{U}$ abundance is greater than $0.1 \%$, as is typically the case for highly enriched uranium (HEU) that has been enriched from recycled reactor fuel. This calculation indicates that SIMS can determine the ${ }^{236} \mathrm{U}$ abundance in recycled HEU with a low relative uncertainty despite the additional contribution due to the hydride correction. Fig. 7 shows a similar plot for $3 \%{ }^{235} \mathrm{U}$ enrichment with the other parameters held the same. Here the absolute uncertainty of the ${ }^{236} \mathrm{U}$ abundance increases from $0.000035 \%$ to $0.00063 \%$ as the relative abundance increases from $0 \%$ to $0.1 \%$, and the relative uncertainty is less than $10 \%$ when the ${ }^{236} \mathrm{U}$ abundance is greater than $0.0006 \%$.

\section{Effect of single vs. multichannel detection}

With the exception of the comparison of the results with Ranebo et al., the examples in this report have been derived with 
the assumption of a single pulse-counting detector and peakswitching. The effect of using the multicollector on the IMS1280 according to this model can be easily determined by applying $100 \%$ counting time to each of the acquired isotopes in the model calculations. Because the single detector calculations assumed $25 \%$ counting time each for $\mathrm{m} / \mathrm{z} 236$ and 239 , the multichannel case results in 4 times greater counts for each of these species, and therefore a factor of 2 lower uncertainty in the net ${ }^{236} \mathrm{U}$ abundance if all other parameters are held the same.

\section{Prospects for reducing the hydride ratio}

Several different approaches may reduce the uranium hydride ion production during SIMS analysis. If the hydrogen source is from the residual vacuum in the sample chamber one may attempt to improve the vacuum in the vicinity of the sample by improved pumping, including use of a cold trap if the source is from residual water vapor. If the hydrogen source is from the sample itself, it might be driven off by heating the sample although this could also alter the chemical form of the uranium. A third potential source is the substrate on which particles are mounted. Pyrolytic graphite or vitreous carbon are commonly used for mounting uranium particles because these materials have high purity, low vapor pressure, and produce no significant high mass polyatomic ions that could create spectral interferences with uranium ions. However, literature reports indicate that high purity graphite may contain as much as $1 \%$ hydrogen that can be reduced by vacuum annealing, but reabsorption will occur when the graphite is exposed to air. ${ }^{7}$ To assess the effect of the substrate on uranium hydride ion production we have investigated the hydride ion signal from uranium oxide particles mounted on graphite as a function of time (ion dose) and compared the results with those obtained from particles mounted on a silicon substrate.

A particle of CRM U900 several micrometers in size, selected from a dispersion of particles on a pyrolytic graphite substrate, was bombarded in an LG-SIMS instrument with an $\mathrm{O}^{-}$primary ion beam of $23 \mathrm{keV}$ impact energy and $30 \mathrm{nA}$ ion current. The beam was focused by Köhler illumination to an oval spot about $50 \mu \mathrm{m} \times 75 \mu \mathrm{m}$ in size centered over the particle. The secondary ion species ${ }^{238} \mathrm{U}^{+}$and ${ }^{238} \mathrm{U}^{1} \mathrm{H}^{+}$were monitored as the particle was eroded by ion sputtering. The pressure in the sample chamber during this analysis was $8.9 \times 10^{-8} \mathrm{~Pa}$.

Fig. 8 shows the results of this measurement, with the ${ }^{238} \mathrm{U}^{+}$ intensity and the ${ }^{238} \mathrm{U}^{1} \mathrm{H}^{+} / 238 \mathrm{U}^{+}$ratio plotted on different axes. The ${ }^{238} \mathrm{U}^{+}$intensity rises, goes through a maximum, and then decreases monotonically as the mass of the particle is eroded by sputtering. The hydride ratio drops quickly from an initial value above $10^{-2}$ and then decreases more slowly to a value approaching $10^{-4}$. In the region where the ${ }^{238} \mathrm{U}^{+}$signal is at a maximum the hydride ratio is about $10^{-3}$.

A similar experiment was conducted by measuring the same two species from a U900 particle on a semiconductor grade silicon substrate. In this case the primary ion current was $50 \mathrm{nA}$

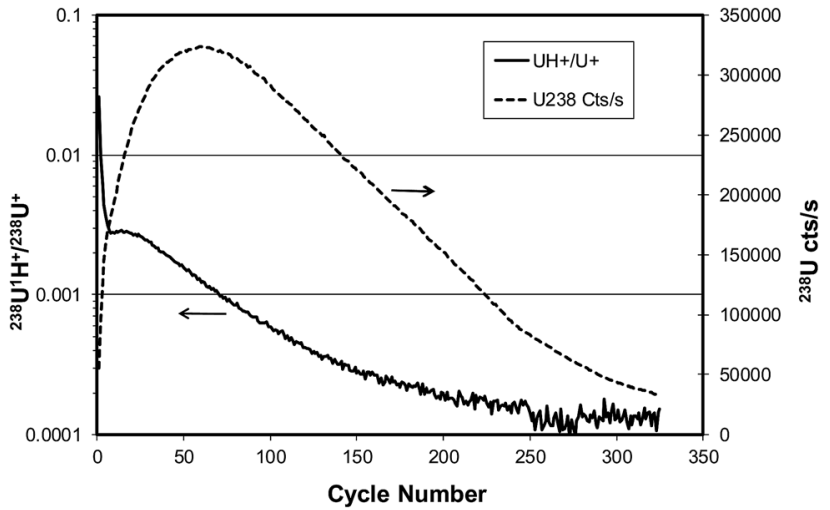

Fig. 8 Evolution of ${ }^{238} \mathrm{U}^{+}$signal (dashed line) and ${ }^{238} \mathrm{U}^{1} \mathrm{H}^{+} /{ }^{238} \mathrm{U}^{+}$ratio (solid line) for $\mathrm{U} 900 \mathrm{U}_{3} \mathrm{O}_{8}$ particle on graphite substrate sputtered by $23 \mathrm{keV} \mathrm{O}^{-}$ion beam.

and the sample chamber pressure was $1.3 \times 10^{-7} \mathrm{~Pa}$. The results shown in Fig. 9 are dramatically different from the graphite case. For the silicon substrate the uranium signal rises rapidly after sputtering begins, then decreases rapidly, then rises to a second maximum and finally decreases as the particle is eroded away. We tentatively attribute the initial drop in the uranium signal to the creation of an $\mathrm{SiO}_{2}$ layer on the silicon after sufficient oxygen has been implanted by the primary ion beam. ${ }^{4}$ This oxide layer may reduce the ion formation probability of the uranium. The overall efficiency of uranium detection between graphite and silicon substrates cannot be deduced from this example because the particle sizes were not known, but comparisons using monodisperse microspheres have shown that the efficiency with silicon is nearly as high as with graphite. ${ }^{4}$ After the onset of sputtering the hydride ratio decreases rapidly to a value of about $10^{-5}$ before the maximum uranium signal is reached and stays approximately at the same ratio as the particle mass is depleted. The residual vacuum in the sample chamber is clearly not the primary determinant of the hydride ratio because the sample chamber pressure is actually higher for the silicon case. Rather, the type of substrate

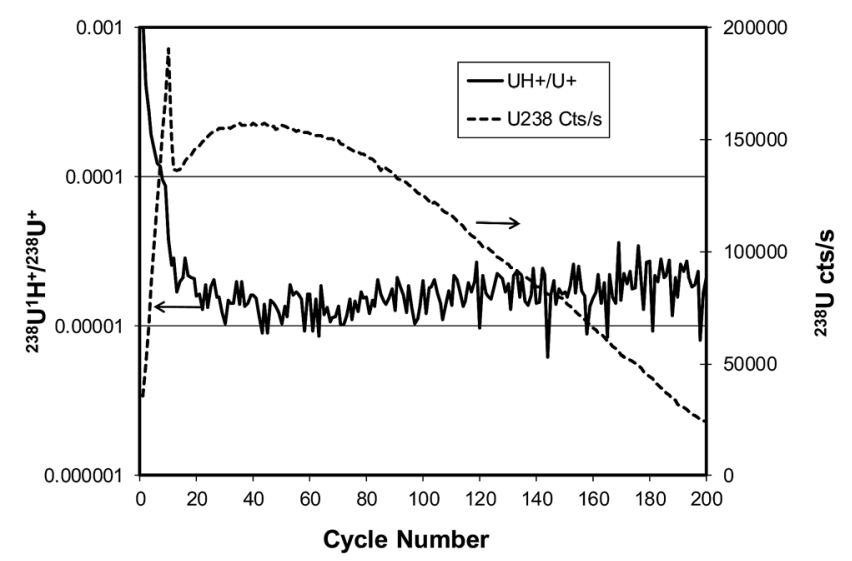

Fig. 9 Evolution of ${ }^{238} \mathrm{U}^{+}$signal (dashed line) and ${ }^{238} \mathrm{U}^{1} \mathrm{H}^{+} /^{238} \mathrm{U}^{+}$ratio (solid line) for $\mathrm{U} 900 \mathrm{U}_{3} \mathrm{O}_{8}$ particle on silicon substrate sputtered by $23 \mathrm{keV} \mathrm{O}^{-}$ion beam. 
is what makes a significant difference. The use of a silicon substrate would therefore be expected to have a substantial effect on the ${ }^{236} \mathrm{U}$ detection limit according to Fig. 2, i.e., a reduction by a factor of 3.2 for a factor of 10 reduction in the hydride ratio. A silicon substrate would also be expected to reduce the ${ }^{236} \mathrm{U}$ uncertainty for cases where the hydride ratio term in eqn (3) and (4) is significant. The effects depicted in Fig. 8 and 9 have been reproduced for multiple particles.

\section{Experimental test of uncertainty model}

The model calculations described above were tested in several experiments in which particles of $\mathrm{U}_{3} \mathrm{O}_{8}$ reference materials were analyzed in an LG-SIMS instrument to measure their isotopic composition and uncertainty. Measurements were made by cycling through the species ${ }^{234} \mathrm{U}^{+},{ }^{235} \mathrm{U}^{+},{ }^{236} \mathrm{U}^{+}+{ }^{235} \mathrm{U}^{1} \mathrm{H}^{+},{ }^{238} \mathrm{U}^{+}$ and ${ }^{238} \mathrm{U}^{1} \mathrm{H}^{+}$and recording the counts from a single electron multiplier detector over 20 cycles of data acquisition while the particle was bombarded with $23 \mathrm{keV} \mathrm{O}^{-}$ions in a static defocused beam that was much larger than the particle (Köhler illumination). For each cycle the ${ }^{236} \mathrm{U}$ ratio to the most abundant isotope was calculated according to eqn (1) or (2), the data were adjusted by time interpolation, corrected with instrumental mass fractionation factors that were determined by calibration with U900 particles, and converted from isotopic ratios to atom percents for each data cycle. The final reported atom percents for a run were the unweighted averages over the 20 cycles, and the reported uncertainties were the standard deviations of the means over the 20 cycles. The ${ }^{236} U$ uncertainty was compared with the model calculation that used as input parameters the known isotopic composition, the fraction of the cycle time over which each species was recorded, the total counts of uranium corrected for duty cycle for that run and the average hydride ratio for that run.

Other sources of background signals at the mass position of ${ }^{236} \mathrm{U}$ were also evaluated to determine if they were significant. The background electronic noise of the pulse-counting detection system was monitored periodically at a mass position where no signal was present and was found to be less than 0.003 counts per $\mathrm{s}$, i.e., less than 1 count in 5 minutes, which should be insignificant for reasonable counting times. The other background source that was considered was the scatter tail from the major uranium peak. Signals from a U900 particle were measured at half-mass positions with respect to ${ }^{234} U^{+}$, then interpolated to the integral mass positions and converted to ratios with respect to the major isotope in this material, ${ }^{235} \mathrm{U}^{+}$. In this way it was determined that the tail of the ${ }^{235} \mathrm{U}^{+}$peak contributes a signal about $5 \times 10^{-8}$ as large as the ${ }^{235} \mathrm{U}^{+}$peak at the $m / z 236$ mass position. By extension of the U900 data, if the major isotope in a particle were ${ }^{238} U$ the contribution from that peak at the $m / z 236$ mass position would be about $6 \times 10^{-8}$ as large as the ${ }^{238} \mathrm{U}^{+}$peak. In either case the tail would contribute a negligible amount to the $m / z 236$ signal under normal particle analysis conditions. Note that these results apply when the single large electron multiplier detector is used on the LG-SIMS. We have not made similar measurements with the multicollector detector which may have a larger peak tail contribution.

In the first experimental example of ${ }^{236} \mathrm{U}$ measurements the same relatively large CRM U900 particle was analyzed sequentially over 9 runs under varying conditions. For the first 6 runs the primary beam current was $0.75 \mathrm{nA}$, for the next 2 runs it was $2 \mathrm{nA}$, and for the final run the current was $5 \mathrm{nA}$. Over this series of runs the hydride ratio monotonically decreased from $3.0 \times 10^{-3}$ to $1.5 \times 10^{-4}$, i.e., by a factor of 20 . The total uranium ion counts corrected for duty cycle ranged from $6.1 \times 10^{7}$ to $1.7 \times 10^{8}$. The measured weighted average ${ }^{236} \mathrm{U}$ isotopic abundance with standard deviation of the mean was $0.3320 \pm 0.0008$ atom percent (certified value $0.3327 \pm 0.0005$ ). The comparison of the measured $v s$. predicted uncertainties for ${ }^{236} \mathrm{U}$ for these 9 measurements is shown in Fig. 10. The measured uncertainties ranged from 0.0052 atom percent in the first run to 0.0014 atom percent in the last run, the decrease largely driven by the decrease in hydride ratio, with the total uranium counts having a secondary effect since they only varied by about a factor of 3 . The measured and predicted uncertainties are well correlated, with a correlation coefficient of 0.95 . These results are also in good agreement with Fig. 5(c) which was calculated for a fixed value of $10^{8}$ uranium ion counts for U900. The figure shows that the ${ }^{236} \mathrm{U}$ uncertainty for a single particle measurement cannot be reduced below 0.001 atom percent under the specified conditions.

In the second example $\mathrm{U}_{3} \mathrm{O}_{8}$ particles of CRM U030a were measured to determine their isotopic compositions on graphite and silicon substrates with an $\mathrm{O}^{-}$primary ion beam under Köhler illumination. This material has a ${ }^{236} \mathrm{U}$ content of 0.000599 atom percent. Each particle was first presputtered for 10 minutes with a $30 \mathrm{nA}$ primary ion beam to stabilize the hydride ratio (see Fig. 8). The isotopic composition was then measured according to the procedure described above. A comparison of the measured vs. predicted uncertainties for particles on the two different substrates is shown in Fig. 11. For

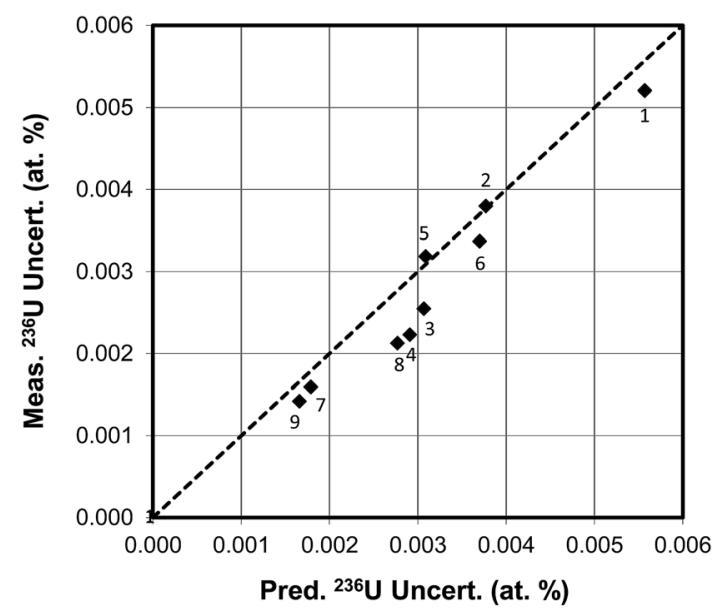

Fig. 10 Measured vs. predicted ${ }^{236} \mathrm{U}$ uncertainty for one CRM U900 particle measured 9 times successively with $O^{-}$primary beam and Köhler illumination. First 6 measurements at $0.75 \mathrm{nA}, 7^{\text {th }}$ and $8^{\text {th }}$ at $2 \mathrm{nA}, 9^{\text {th }}$ at $5 \mathrm{nA}$. Certified ${ }^{236} \mathrm{U}$ abundance is 0.3327 atom percent. 


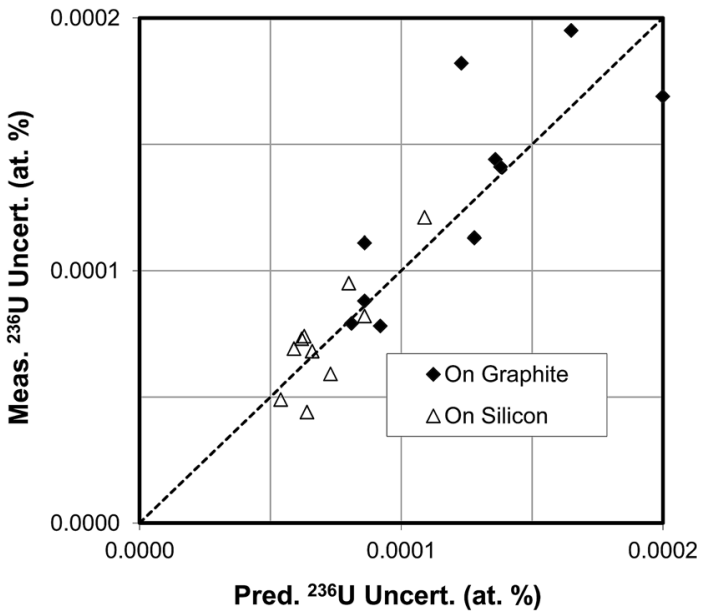

Fig. 11 Measured vs. predicted ${ }^{236} U$ uncertainty for different CRM U030a particles on graphite and on silicon substrates measured with $\mathrm{O}^{-}$primary beam and Köhler illumination. Each particle was presputtered for 10 minutes at $30 \mathrm{nA}$ to reduce hydride ratio. Certified ${ }^{236} \mathrm{U}$ abundance is 0.000599 atom percent.

the particles on graphite substrates the average total uranium counts were $1.2 \times 10^{8}$, the average hydride ratio was $8.1 \times 10^{-4}$, and the average uncertainty was 0.000135 atom percent. For the particles on silicon substrates the average total uranium counts were also $1.2 \times 10^{8}$, the average hydride ratio was $7.5 \times 10^{-5}$, a factor of about 11 lower, and the average uncertainty was 0.000073 atom percent, a factor of about 2 lower. The correlation between measured and predicted uncertainties is not as tight as in the CRM U900 case, but this is to be expected because
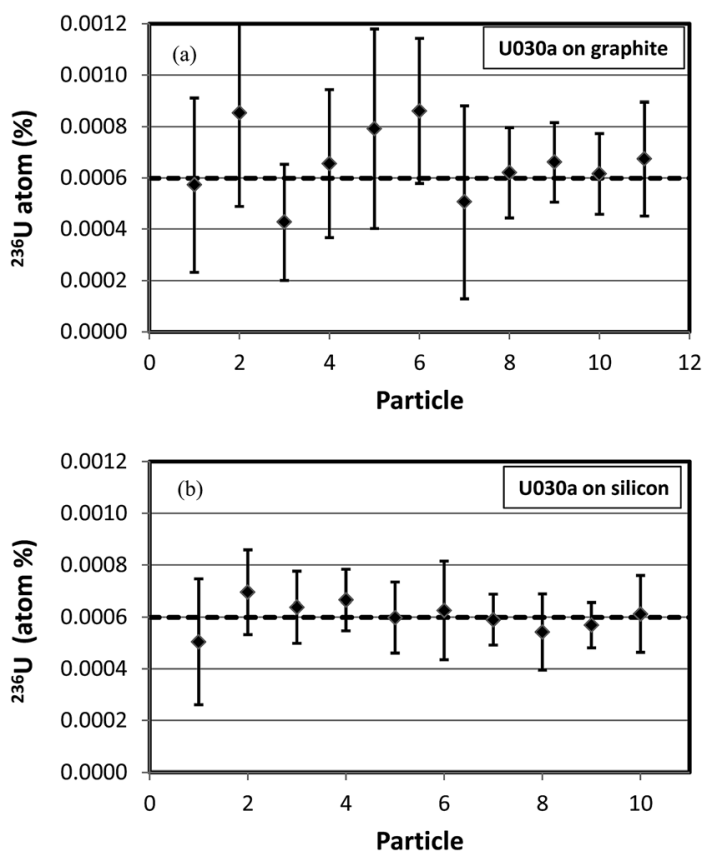

Fig. 12 Measured ${ }^{236} \mathrm{U}$ abundances in CRM U030a particles on graphite (a) and silicon (b) substrates. Error bars are 2 standard deviations. Dashed line is certified value of 0.000599 atom percent. the net counts of ${ }^{236} \mathrm{U}^{+}$are quite small for U030a. These results are in good agreement with Fig. 5(b) which also shows that the ${ }^{236} \mathrm{U}$ uncertainty cannot be reduced below 0.00005 atom percent under the specified conditions, no matter how small the hydride ratio. The results are presented in a different way in Fig. 12(a) and (b) where the ${ }^{236} \mathrm{U}$ atom percent values are plotted for each particle measurement and the error bars are two standard deviations. In all cases the error bars overlap the certified value of 0.000599 atom percent. These figures graphically illustrate the factor of two reduction in uncertainty for the silicon substrate as a result of the lower hydride ratio.

\section{Conclusion}

The preceding model calculations are idealized since they assume that the only variability in the measurement of the ${ }^{236} \mathrm{U}$ abundance is produced by the inherent and inevitable statistical fluctuations in ion counting. In reality other contributions, for example instability in the primary ion source, may have an additional measureable effect. In general, these other contributions only become significant when the Poisson contribution to the relative uncertainty is less than $1 \%$. The presence of these additional contributions can be tested in real-world measurements by comparing the Poisson-only prediction based on total ion counts of each species with the cycle-by-cycle variability through the use of the F statistic, for example.

The general conclusions of this study are as follows:

- If a $1 \mu \mathrm{m}$ particle of pure uranium oxide that contains a significant amount of ${ }^{236} \mathrm{U}$ is completely consumed, a large geometry SIMS can measure the abundance of ${ }^{236} \mathrm{U}$ with relatively small uncertainty at any enrichment level. If no ${ }^{236} \mathrm{U}$ is present the SIMS measurement can establish its absence (i.e., less than $0.0001 \%$ ) under typical experimental conditions with a graphite substrate only if the ${ }^{235} \mathrm{U}$ enrichment level is less than $3 \%$.

- Changing to a substrate that produces lower hydride ratios than does graphite will allow a detection limit of $0.0001 \%$ to be achieved for ${ }^{236} \mathrm{U}$ at a higher enrichment level in a $1 \mu \mathrm{m}$ particle, for example up to $20 \%$ enrichment if a hydride ratio of $10^{-5}$ can be achieved. For a silicon substrate with an $\mathrm{O}^{-}$primary ion beam the rapid $\mathrm{U}^{+}$signal changes exhibited in Fig. 9 could be problematic for precise isotopic analysis if peak-switching is used. However, the same signal transients have not been observed under $\mathrm{O}_{2}{ }^{+}$bombardment, ${ }^{4}$ which may be a more suitable analytical condition for use with a silicon substrate.

- SIMS, when practiced as described here, cannot establish the absence (detection limit $0.0001 \%$ ) of ${ }^{236} \mathrm{U}$ in highly enriched uranium in a particle smaller than several micrometers in size. This is a direct consequence of the inevitable presence of an interfering uranium hydride ion peak and the statistics of ion counting. For example, as an extrapolation of Fig. 3, a hydride ratio below about $2 \times 10^{-7}$ would be required to achieve this detection limit in a $93 \%$ enriched particle with $10^{10}$ uranium atoms based on the model presented. This hydride ratio is nearly a factor of 100 lower than the value achieved in Fig. 9 with a silicon substrate. 


\section{References}

1 Y. Ranebo, et al., Improved isotopic SIMS measurements of uranium particles for nuclear safeguard purposes, J. Anal. At. Spectrom., 2009, 24, 277-287.

$2 \mathrm{X}$. Wang, et al., ${ }^{236} \mathrm{U}$ measurement with accelerator mass spectrometry at CIAE, Nucl. Instrum. Methods Phys. Res., Sect. B, 2010, 268, 2295-2299.

3 New Brunswick Laboratory, U.S. Department of Energy, Certificates of Analysis available at http://science.energy.gov/ $\mathrm{nbl} /$ certified-reference-materials/prices-and-certificates/ uranium-certified-reference-materials-price-list/, accessed October 2016.
4 N. Sharp, J. D. Fassett and D. S. Simons, Uranium ion yields from monodisperse uranium oxide particles, J. Vac. Sci. Technol., B: Nanotechnol. Microelectron.: Mater., Process., Meas., Phenom., 2016, 34, 03H115.

5 D. S. Simons, Propagation of Errors in the Calculation of Atomic Percents from Isotopic Ratios, unpublished report, 2000.

6 F. Schaefer, P. D. P. Taylor, S. Valkiers and P. De Bievre, Computational procedures for the treatment of measured or published isotope abundance data, Int. J. Mass Spectrom. Ion Processes, 1994, 133, 65-71.

7 R. Bastasz, Residual hydrogen concentration levels in graphite, J. Vac. Sci. Technol., A, 1988, 6, 2116-2118. 